\title{
Leishmania (Leishmania) mexicana en el corregimiento de San Matías, municipio de Gómez Plata, Antioquia, Colombia
}

\author{
Diana Sierra, Marcela Ochoa, José Ignacio Calle, Gisela García, \\ Diana Colorado, Iván Darío Vélez \\ Programa de Estudio y Control de Enfermedades Tropicales, PECET, \\ Universidad de Antioquia, Medellín, Colombia.
}

\begin{abstract}
Introducción. La leishmaniaisis es una enfermedad encontrada en focos naturales de infección donde están presentes insectos vectores y mamíferos reservorios de Leishmania.

Objetivo. Registrar por primera vez la presencia de Leishmania (Leishmania) mexicana Biagi, 1953, en el corregimiento de San Matías, municipio de Gómez Plata, departamento de Antioquia. Materiales y métodos. La especie fue aislada de un paciente con leishmaniasis cutánea localizada e identificada por la técnica de Inmunofluorescencia utilizando anticuerpos monoclonales específicos de especie y electroforesis de enzimas .

Resultados y conclusión. Su perfil isoenzimático similar al de las cepas de referencia $L$. (L.) mexicana (MNCY/BZ/62/M379) y L. (L.) mexicana (MHOM/BE/82/BEL21), permitió concluír que la especie aislada del paciente es $L$. (L.) mexicana.
\end{abstract}

Palabras clave: leishmaniasis, Leishmania, Colombia.

Leishmania (Leishmania) mexicana in the village of San Matias, municipality of Gomez Plata, North West of Antioquia, Colombia

Introduction. Leishmaniasis is found in natural foci of infection where both sand fly vectors and mammalian reservoirs of Leishmania are present.

Objective. To report for the first time the presence of Leishmania (Leishmania) mexicana Biagi, 1953, in the village of San Matías, municipality of Gomez Plata, Department of Antioquia.

Materials and methods. The parasite was isolated from a patient with localized cutaneous leishmaniasis and was identified by the immunofluorescence technique using specific monoclonal antibodies against $L$. mexicana species and isoenzyme electrophoresis.

Results and conclusion. The isoenzymatic profiles of the reference strains $L$. (L.) mexicana (MNCY/BZ/62/M379) and L. (L.) mexicana (MHOM/BE/82/BEL21) were similar to that of the isolate recovered from the patient, allowing us to classify it as $L$. (L.) mexicana.

Key words: leishmaniasis, Leishmania, Colombia.

La leishmaniasis es un conjunto de enfermedades, causadas, al menos, por 20 especies de parásitos del género Leishmania, transmitidos a los mamíferos a través de la picadura de insectos hembras hematófagas pertenecientes a los

\footnotetext{
Correspondencia:

Diana María Sierra, Programa de Estudio y Control de Enfermedades Tropicales, PECET, Universidad de Antioquia. Sede de Investigaciones Universitaria, SIU, Laboratorio 632; Calle 62 № 52-59. Medellín, Colombia.

Teléfono: (574) 210 6502; fax: (574) 2106511 dsierra68@yahoo.com
}

Recibido: 1/07/05; aceptado: 24/02/06 géneros Lutzomyia en el Nuevo Mundo y Phlebotomus en el Viejo Mundo; los reservorios son animales domésticos y silvestres que albergan el parásito, lo cual permite que los vectores se infecten de ellos y persista el ciclo de transmisión (1).

Los parásitos de Leishmania están agrupados en dos subgéneros, Viannia y Leishmania. El primero incluye las especies propias del continente americano como L. (V.) peruviana, Vélez 1913, L. (V.) guyanensis, Floch 1954, L. (V.) panamensis, Lainson y Shaw 1972 y L. (V.) braziliensis que son las especies más ampliamente distribuidas 
en Colombia y responsables del mayor número de casos de leishmaniasis cutánea y mucosa (2). En el subgénero Leishmania se encuentran especies distribuidas tanto en el Viejo Mundo (Europa, Asia y África) como en América (3). En este último subgénero se encuentra $L$. (L.) mexicana especie que se distribuye desde el sur de los Estados Unidos hasta Colombia, la cual produce generalmente úlceras cutáneas pequeñas, principalmente en cara y pabellón auricular. En Colombia esta especie ha presentado una distribución limitada, encontrándose en los departamentos de Caldas, Putumayo, Nariño y Santander (4-6).

Según la especie de Leishmania, se desarrollan las diferentes formas clínicas de la enfermedad como leishmaniasis cutánea, causada por especies de Leishmania de los subgéneros Viannia y Leishmania. Se distribuye desde el sur de los Estados Unidos hasta el norte de Argentina (7).

En el presente trabajo se registra por primera vez el hallazgo de $L$. (L.) mexicana en el corregimiento de San Matías, municipio de Gómez Plata, departamento de Antioquia.

\section{Materiales y métodos}

\section{Área de estudio}

El corregimiento de San Matías esta localizado a $6^{\circ} 40^{\prime} 43^{\prime \prime} \mathrm{N}-75^{\circ} 13^{\prime} 01^{\prime \prime} \mathrm{O}$. Su altura sobre el nivel del mar es de 1.800 metros sobre el nivel del mar (m.s.n.m), con temperatura promedio de $20^{\circ} \mathrm{C}$; está ubicado entre los ríos Guadalupe y Porce.; es de área montañosa con zonas planas y valles y elevaciones pertenecientes a la Cordillera Central; su economía depende básicamente de cultivos (caña panelera, yuca, café, maíz y plátano), ganadería y minería.

El paciente con leishmaniasis cutánea localizada residente de esta localidad fue examinado durante un estudio de prevalencia de leishmaniasis realizado en el corregimiento de San Matías.

\section{Búsqueda activa de casos, aislamiento e identificación de la cepa de Leishmania sp.}

En la finca El Loro del corregimiento de San Matías, se presentaron en los últimos seis meses -por primera vez en la historia del municipio- tres casos autóctonos de leishmaniasis cutánea en trabajadores de la finca, los cuales fueron diagnosticados mediante examen directo en el Laboratorio Clínico del Hospital de Santa Isabel.

En el momento de nuestro estudio, a uno de estos pacientes con lesiones se le tomó muestra por aspirado del borde activo de la lesión y el material obtenido se sembró en el medio de cultivo NovyMcNeal- Nicolle (NNN). Posteriormente, se realizó un subcultivo en masa en el medio NNN modificado para proceder a la identificación de los parásitos por inmunofluorescencia indirecta (IFI), utilizando anticuerpos monoclonales específicos de especie (8) y su posterior confirmación por isoenzimas (9).

Para esta técnica se usaron las enzimas glucosa -6-fosfato deshidrogenasa, fosfogluconato deshidrogenasa, superóxido dismutasa, fosfoglucosa mutasa, nucleósido hidrolasa, manosa-fosfato isomerasa, glucosa-fosfato isomerasa de las cepas de referencia de la Organización Mundial de la Salud, HOM/BR/75/ M2903 (L. (V.) braziliensis), HOM/Br/75/M4147 (L. (V.) guyanensis, HOM/PA/71/LS94 (L. (V.) panamensis), IFLA/BR/67/PH8(L. (L) amazonensis, $\mathrm{MNCY/BZ/62/M379} \mathrm{(L.} \mathrm{(L.)} \mathrm{mexicana),} \mathrm{MHOM/}$ BE/82/BEL21 (L. (L.) mexicana) y MHOM/VE/74/ PMH3 (L. (L.) venezuelensis).

\section{Consideraciones éticas}

Los procedimientos realizados fueron aprobados por el Comité de Bioética de la Corporación Académica para el Estudio de Patologías Tropicales de la Universidad de Antioquia, ya que cumple con los requisitos establecidos en la Resolución № 008430 de 1993, sobre normas científicas, técnicas y administrativas para la investigación en salud.

\section{Resultados}

El paciente analizado en el corregimiento de San Matías presentó lesiones cutáneas activas, lo que permitió la toma de muestra para el cultivo y el posterior aislamiento del parásito para su identificación.

La técnica de IFI utilizando anticuerpos monoclonales específicos de especie, permitió establecer, en primera instancia, que el parásito 
pertenecía al complejo mexicana y, posteriormente, su perfil isoenzimático similar al de las cepas de referencia $L$. (L.) mexicana (MNCY/ BZ/62/M379) y L. (L.) mexicana (MHOM/BE/82/ BEL21), permitió concluir que la especie aislada del paciente es $L$. ( $L$ ). mexicana.

\section{Discusión}

En Colombia se han informado brotes epidémicos de leishmaniasis desde 1986 (10) y desde entonces el Programa de Estudio y Control de Enfermedades Tropicales, PECET, ha venido realizando estudios de campo en varias regiones del país como Montebello, Antioquia (1984), San Roque, Antioquia (1987), Saiza, Córdoba (1990), Arboletes, Antioquia (1997), Yalí, Maceo y Cisneros, Antioquia en 2002 (informes internos PECET); con excepción de Montebello la especie responsable fue una variante enzimática de $L$. (V.) braziliensis, muy emparentada por isoenzimas con L. (V.) peruviana, (Vélez 1913); para los otros focos la especie responsable fue $L$. (V.) panamensis la cual presenta una amplia distribución en Colombia (4).

El género Leishmania comprende 30 especies, las cuales presentan distribución ecológica y geográfica diferente y pueden infectar gran variedad de reservorios y vectores $(11,12)$. Para el diseño de medidas de prevención y control de la enfermedad es importante hacer una correcta identificación de la especie de parásito que está causando la enfermedad en un área dada.

Nuestro estudio confirma la transmisión activa de Leishmania causante de manifestaciones clínicas cutáneas en el corregimiento de San Matias. Esta afirmación se respalda por los casos humanos detectados en la zona y, también, por el aislamiento, aunque sólo en un paciente de $L$. (L.) mexicana que ocasiona formas clínicas cutáneas y de la cual en Colombia se ha informado su presencia en los departamentos de Caldas, Putumayo, Nariño y Santander (4-6).

Igualmente, es importante la presencia de Lutzomyia columbiana del grupo Verrucarum (Ristorcelly \& Van Ty, 1941) (no se presentan los resultados), especie de importancia médica y que fue incriminada como vector de bartonelosis en el sur de Colombia entre los años 1930 y 1940 (13) y, más recientemente, asociada a la transmisión de $L$. (L.) mexicana en Samaniego, Nariño (5).

Es importante que las autoridades de salud presten una vigilancia constante por la posibilidad de la aparición de nuevos casos de leishmaniasis en esta localidad. Además, es necesario realizar estudios epidemiológicos que incluyan la búsqueda de la infección tanto en humanos como en reservorios y flebótomos, elementos que constituyen la triada epidemiológica de leishmaniasis.

\section{Agradecimientos}

Agradecemos al personal del Hospital de Santa Isabel por su participación y colaboración; a los habitantes de la localidad de San Matías y a Imerio Correa de la finca EI Loro por toda su cooperación para la ejecución de este estudio.

\section{Financiación}

Este estudio fue realizado dentro del convenio interadministrativo realizado entre la Dirección Seccional de Salud de Antioquia y el Programa de Estudio y Control de Enfermedades Tropicales, PECET, de la Universidad de Antioquia; contrato código № $\mathrm{Cl}$ 217-2002.

\section{Conflicto de intereses}

Los autores manifiestan que no existe conflicto personal ni financiero de interés con otras personas $u$ organizaciones que pudieran influenciar inadecuadamente los datos presentados en esta revisión.

\section{Referencias}

1. Organización Mundial de la Salud. The leishmaniases. Technical report series 701. Ginebra: OMS; 1984.p.140.

2. Rioux JA, Lanotte G, Serres E, Pratlong F, Bastien $\mathbf{P}$, Périères $\mathbf{J}$. Taxonomy of Leishmania. Use of isoenzymes. Suggestions for a new classification. Ann Parasitol Hum Comp 1990;65:111-25.

3. Thomaz-Soccol V, Lanotte G, Rioux JA, Pratlong F, Martini-Dumas A, Serres E. Phylogenetic taxonomy of New World Leishmania. Ann Parasitol Hum Comp 1993;68:104-6.

4. Corredor A, Kreutzer RD, Tesh RB, Boshell J, Palau MT, Cáceres E et al. Distribution and etiology of 
leishmaniasis in Colombia. Am J Trop Med Hyg 1990; 42:206-14.

5. Montoya-Lerma J, Cadena H, Segura I, Travi B. Association of Lutzomyia columbiana (Diptera: Psychodidae) with a leishmaniasis focus in Colombia due to species of the Leishmania mexicana complex. Mem Inst Oswaldo Cruz 1999;94:277-83.

6. Rodríguez G, Corredor A, Cáceres E, Cassiano G, Arroyo C, Palau M et al. Leishmaniasis difusa. Biomédica 1985;5:95-111.

7. Organizacion Panamericana de la Salud. Epidemiología y control de las leishmaniasis en las Américas, por país o territorio. Cuaderno técnico No. 44. Washington: OPS; 1996.p. 62.

8. McMahon-Pratt D, David JR. Monoclonal antibodies that distinguish between New World species of Leishmania. Nature 1981;291:581-3.

9. Miles MA. Identificación bioquímica de las leishmanias. Bol Oficina Sanit Panam 1986;101:217-29.
10. Vélez ID, Wolff M, Valderrama R, Escobar JP, Osorio L. Comunity and environmental risk factors associated with cutaneus leishmaniosis in Montebello, Antioquia, Colombia. En: IDRC. Leishmaniosis control strategies: a critical evaluation of IDRC-supported research. Mérida: DF IDRC Publications; 1991. p.261-74.

11. Grimaldi Jr G, Tesh RB, McMahon-Pratt D. A review of the geographic distribution and epidemiology of leishmaniasis in the New World. Am J Trop Med Hyg 1989;41:687-725.

12. Grimaldi Jr G, Momen H, Naiff RD, McMahon-Pratt D, Barrett TV. Characterization and classification of leishmanial parasites from humans, wild mammals, and sand flies in the Amazon region of Brazil. Am J Trop Med Hyg 1991;44:645-61.

13. Rozeboom LE. The identity of the Phlebotomus associated with bartonellosis in Colombia. Ann Entomol Soc Am 1947;40:705-14. 